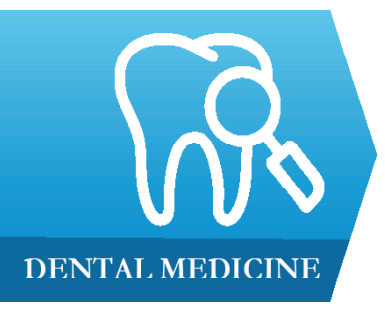

1) Oral and Maxillofacial Pathology Department, KSR Institute of Dental Science \& Research, Tamilnadu, India

2) Oral and Maxillofacial Pathology Department, Vivekanandha Dental College for Women, Tamilnadu, India

DOI: $10.15386 / \mathrm{mpr}-1686$

Manuscript received: 28.04 .2020

Received in revised form: 14.05 .2020

Accepted: 30.05.2020

Address for correspondence:

chintuchnth@gmail.com

This work is licensed under a Creative Commons Attribution-NonCommercialNoDerivatives 4.0 International License

\title{
Evaluation of gingival and periodontal status in obese and non-obese type II diabetic patients - a cross sectional study
}

\author{
Kenniyan Kumar SriChinthu ${ }^{1}$, Velusamy Pavithra ${ }^{1}$, G.S. Kumar ${ }^{1}$, \\ Harikrishnan Prasad ${ }^{1}$, Perumal Prema ${ }^{1}$, \\ Thuckanickenpalayam Ragunathan Yoithapprabhunath ${ }^{2}$, \\ Nagarajan Rangarajan ${ }^{2}$
}

\begin{abstract}
Objective. The aim of this study was to evaluate gingival and periodontal status in obese and non-obese type II Diabetic Patients.

Methods. The study population comprised of 75 subjects visiting the outpatient department of our institution, divided into three different groups, group 1 (obese diabetic), group 2 (non-obese diabetic), and group 3 (obese, non-diabetic). Diabetic status was assessed with $\mathrm{HbA1c}$ values and obesity status was assessed by body mass index (BMI) score greater than or equal to $30 \mathrm{~kg} / \mathrm{m}^{2}$. Gingival and periodontal status were assessed using the Gingival Index (GI) and Community Periodontal Index (CPI) respectively.

Results. The mean gingival index score in group 1, group 2, and group 3 were $1.58,1.54$, and 1.25 , respectively. Gingival status was poor among obese and nonobese diabetic subjects (Groups 1 and 2) when compared with obese non-diabetic patients (Group 3). The periodontal status showed that periodontal pockets were increased in diabetic obese group (15.4\%), followed by diabetic non obese (4.66\%), and non-diabetic obese ( $2 \%$ ) group respectively and loss of attachment was severe in diabetic obese group (60.7\%), followed by diabetic non obese (45.9\%) and nondiabetic obese (15.3\%) respectively.

Conclusion. Gingival and periodontal status was poor in the obese diabetic group compared to non-obese diabetic and obese non diabetic group. Hence, the risk of gingivitis and periodontitis in obese diabetic patients should be addressed earlier to prevent further complications and achieve a good oral health status.
\end{abstract}

Keywords: gingivitis, periodontitis, obesity, diabetes mellitus

\section{Introduction}

Type II Diabetes mellitus (DM) is a universal disorder which affects the quality of life by deregulating many systemic functions in human beings. Its incidence and prevalence is rising in both developed as well as in developing nations like India. DM affects more than 415 million people worldwide and 69 million people in India and is predicted to affect 592 million people by 2035 [1]. It is a common chronic, endocrine and metabolic disorder which is characterized by an abnormal increase in blood glucose levels, insulin resistance, and relative insufficiency of insulin leading to impaired metabolism of carbohydrates, proteins and lipids. This disorder is accompanied by long-term complications such as retinopathy, nephropathy, neuropathy, cardiovascular disorders, and various oral manifestations such as altered salivary flow, mucosal drying and cracking, burning mouth, candidiasis, cheilosis, dental caries, gingivitis, and progressive periodontitis. Periodontitis is 
known as the sixth complication of diabetes mellitus and it increases the response to bacterial plaque by gingiva, due to increased amount of glucose in gingival crevicular fluid (GCF), deficient neutrophil function, and altered collagen metabolism [1-3].

Gingivitis is the initial stage of periodontal disease in which inflammation is limited to gingiva and is usually determined by gingival bleeding and swollen gums. If it is not properly or promptly treated, this may progress to periodontitis. Periodontitis is a chronic inflammatory disease characterized by the destruction of supporting structures of the teeth such as periodontal ligament and alveolar bone. Severe periodontitis has a prevalence of $10 \%$ to $15 \%$ in the general population $[1,4]$.

Apart from periodontitis, there is substantial evidence that a relationship between obesity and type II diabetes exists. Obesity is a multifactorial disease and is becoming a worldwide epidemic with an increasing prevalence in recent years, in both adults as well as in children. Obesity is defined to have excessive amount of body fat. Overweight and obese individuals are defined by measures of weight and height that provide an index of one's mass, referred to as a body mass index (BMI). Currently, BMI is considered to be the most popular indicator for obesity because of its simple and noninvasive procedure. The BMI score of $30 \mathrm{~kg} / \mathrm{m}^{2}$ or greater than $30 \mathrm{~kg} / \mathrm{m}^{2}$ is indicative of obesity [5]. According to the Global Burden of Disease Study 2015 (GBD, 2017), 603.7 million adults and 107.7 million children were obese worldwide. An analysis of the Third National Health and Nutrition Examination Survey (NHANES III) showed that body mass index (BMI) was significantly associated with periodontal disease. It is thought that hormones and cytokines derived from immune cells in adipose tissue cause a series of inflammatory reactions that cause periodontal disease $[4,6]$.

There is therefore a triangular relationship between periodontitis, obesity and diabetes [7]. Hence the rationale of this study was to evaluate the gingival and periodontal status in obese and non-obese type II diabetic patient.

\section{Methods}

The study sample consisted of 75 subjects visiting the outpatient department of our institution, who were categorized into 3 groups of 25 subject each as follows: patients,

Group 1 - Obese (BMI $\geq 30)$ type II diabetic patients,

Group 2 - Non obese $($ BMI <30) type II diabetic

Group 3 - Obese (BMI $\geq 30$ ), non-diabetic patients.

Only individuals with a known history of diabetes for $>5$ year were included in groups 1 and 2. Obesity was defined as BMI $\geq 30 \mathrm{~kg} / \mathrm{m}^{2}$. Patients with a diabetic history of $<5$ years, those under medication for any other systemic disease, those who were habituated to smoking and alcohol intake were excluded from the study. Institutional Ethical Committee clearance was obtained (IEC no. 227/KSRIDSR/EC/2019) prior to commencement of the study. All participating subjects were explained in local vernacular about study procedure and due consent was obtained. Hemoglobin A1c (HbAlc) was estimated in the study population to confirm their diabetic status. After this, the gingival status was assessed using Gingival index following Loe and Silness (1963) criteria by using a mouth mirror and a graduated Williams periodontal probe. The periodontal status was assessed using Community Periodontal Index (CPI) following the Joint Working Committee of WHO / FDI 1997 criteria by using a mouth mirror and CPITN-C probe / WHO probe. The gingival and periodontal investigations were carried out under the close observation of a periodontist. Obesity was assessed by means of Body Mass Index (BMI). BMI was calculated as the ratio of weight in kilograms to the square of height in meters following World Health Organization (WHO) guidelines. The obtained data were analyzed by $\mathrm{Chi}$ square test, one-way ANOVA and Tukey's post hoc using statistical package for social studies (SPSS, version 16 for Windows).

\section{Results}

Table I depicts the comparison of gingival index score across the study population; Gingival inflammation was higher among obese and non-obese diabetic patients (Group 1 and Group 2) than obese non-diabetic patients (Group 3). One-way ANOVA test showed that the difference in Gingival Index score was statistically significant (Figure 1).

Table I. Comparison of Gingival Index Score across the study population.

\begin{tabular}{l|c|c|c} 
Group & Mean & SD & p value \\
\hline Group 1 & 1.58 & 0.30 & \\
Group 2 & 1.54 & 0.28 & .000 \\
Group 3 & 1.25 & 0.23 & $(\mathrm{p}<0.01)$
\end{tabular}

One-way ANOVA test $(\mathrm{p}<0.01-$ Highly Significant $)$

\section{GINGIVAL INDEX}

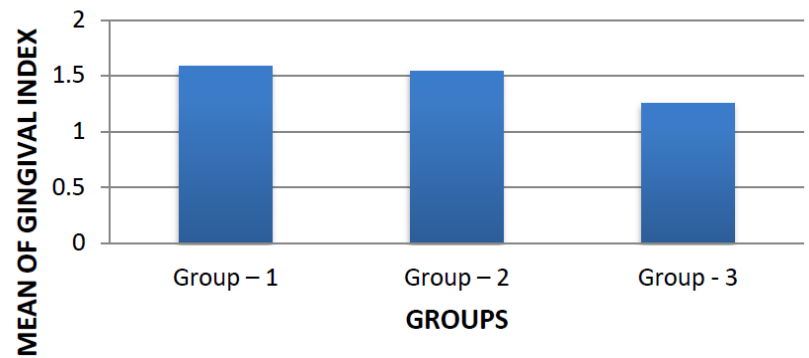

Figure 1. Comparison of Gingival Index Score across the study population. 
Table III. Comparison of Community Periodontal Index Score among the study population.

\begin{tabular}{l|c|c|c|c|c|c|c} 
CPI Score & Healthy (0) & Bleeding (1) & Calculus (2) & Shallow Pocket (3) & Deep Pocket (4) & Excluded (X) & P value \\
Group 1 & $0(0 \%)$ & $32(21.33 \%)$ & $95(63.43 \%)$ & $20(13.4 \%)$ & $3(2 \%)$ & $0(0 \%)$ & .00 \\
Group 2 & $0(0 \%)$ & $48(32 \%)$ & $92(61.33 \%)$ & $3(2 \%)$ & $4(2.66 \%)$ & $3(2 \%)$ & $(\mathrm{p}<0.01)$ \\
Group 3 & $8(5.33 \%)$ & $75(50 \%)$ & $64(42.66 \%)$ & $3(2 \%)$ & $0(0 \%)$ & $0(0 \%)$
\end{tabular}

Chi-Square Test $(\mathrm{p}<0.01-$ Highly Significant)

Table IV. Comparison of Loss of Attachment among the study population.

\begin{tabular}{|c|c|c|c|c|c|c|c|}
\hline CPI Score & 0 [LOA 0 to $3 \mathrm{~mm}$ ] & 1 [LOA 4 to $5 \mathrm{~mm}$ ] & 2 [LOA 6 to $8 \mathrm{~mm}$ ] & 3 [LOA 9 to $12 \mathrm{~mm}$ ] & $4[\mathrm{LOA}>12 \mathrm{~mm}]$ & Excluded (X) & p value \\
\hline Group 1 & $59(39.3 \%)$ & $46(30.7 \%)$ & $34(22.7 \%)$ & $10(6.7 \%)$ & $1(0.6 \%)$ & $0(0 \%)$ & \multirow{3}{*}{$\begin{array}{c}.00 \\
(p<0.01)\end{array}$} \\
\hline Group 2 & $77(51.3 \%)$ & $41(27.3 \%)$ & $25(16.6 \%)$ & $4(2 \%)$ & $0(0 \%)$ & $3(2 \%)$ & \\
\hline Group 3 & $127(84.7 \%)$ & $19(12.7 \%)$ & $3(2 \%)$ & $1(0.6 \%)$ & $0(0 \%)$ & $0(0 \%)$ & \\
\hline
\end{tabular}

Chi-Square Test $(\mathrm{p}<0.01-$ Highly Significant $)$

Tukey's post hoc test showed that there was no statistically significant difference in gingival inflammation between obese and non-obese diabetic patients, while a significant difference existed between non-diabetic and diabetic patients (Table II).

Table II. Comparison of Gingival Index Score among different study groups.

\begin{tabular}{l|c} 
Test Variable & p-value \\
Group 1 Vs Group 2 & $0.98(\mathrm{p}<0.01)$ \\
Group 1 Vs Group 3 & $0.00(\mathrm{p}<0.01)$ \\
Group 2 Vs Group 3 & $0.00(\mathrm{p}<0.01)$
\end{tabular}

Tukey's Post Hoc Test $(\mathrm{p}<0.01-$ Highly Significant $)$

Table III depicts the comparison of Community Periodontal Index Score among the study population. None of the study subjects in Group 1 and Group 2 had healthy periodontium, and there was a higher amount of calculus deposits in Group 1 and Group 2 patients than in Group 3. Group 1 patients showed periodontal pockets in more sextants than Group 3 and Group 2 patients (Figure 2).

\section{COMMUNITY PERIODONTAL INDEX}

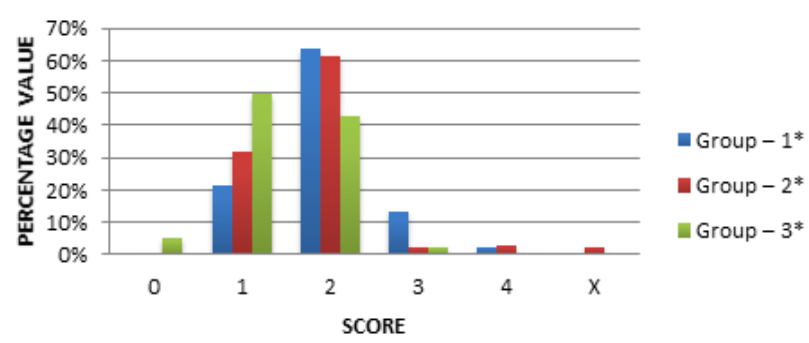

Figure 2. Comparison of Community Periodontal Index Score among the study population.
Table IV depicts the comparison of Loss of Attachment among the study population; more than $50 \%$ of the diabetic patients had loss of attachment. In contrast only $16 \%$ of non-diabetic patients had loss of attachment. Severe loss of attachment was found among obese diabetic patients. Chi-square test showed that there was a significant difference in the LOA score among the study population (Figure 3).

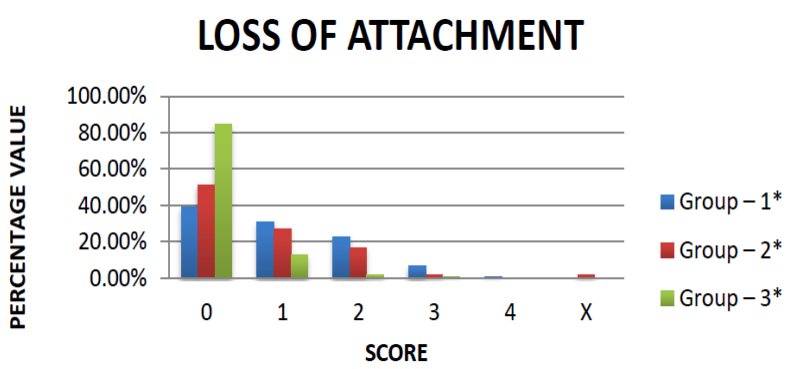

Figure 3. Comparison of Loss of Attachment among the study population.

\section{Discussion}

Type II DM, previously known as adult-onset diabetes, is characterized by high blood sugar level, resistance to insulin and insufficiency of insulin. The lack of insulin affects the metabolism of carbohydrate, protein, and fat and causes a significant disturbance of water and electrolyte homeostasis. Type II DM is recognized as one of the major chronic health problems faced by the world today. It is considered to be one of the most common endocrine and metabolic disorders whose complications reduce life expectancy and is ranked 7 th among the leading causes of death [2]. India was declared as the capital for 
diabetes because 41 million Indians have diabetes to this date and every fifth diabetic in the world is reported to be an Indian [8]. The global prevalence is rapidly increasing, and represents a significant burden to human health because of its numerous complications. Among these, periodontitis is one of the most prevalent oral complications in patients with type 2 diabetes mellitus [9]. In 1993, Harald Löe, a periodontologist, described periodontitis as the sixth complication of diabetes mellitus [7].

Periodontal diseases include destruction of gingival and periodontal (cementum, periodontal ligament, and alveolar bone) structures which lead to gingivitis and periodontitis. In gingivitis, the inflammation is confined only to the gingival tissue, and with good oral hygiene practice it can be reversed. But in periodontitis, the inflammation extends into the deeper structure and results in tissue destruction and resorption which leads to mobility and loss of teeth [10]. It is generally stated that diabetes alone will not give rise to gingival or periodontal disease, but it favours the modification of the gingival periodontal sphere, by increasing the response to bacterial plaque accumulation [11].

Hyperglycemia in diabetes mellitus results in increased advanced glycation end products, thereby accelerating various pro-inflammatory effects which affects various body systems, including the periodontal tissues.

One of the main risk factors for diabetes mellitus is obesity. There is substantial evidence linking obesity, and diabetes. Diabetic individuals are at a higher risk of becoming obese due to insulin resistance of the muscle cells and islet alpha cells. This insulin resistance results in increased hepatic glucose production and insulin levels which are the causes of obesity. Obesity is defined to have excessive amount of body fat. Currently, BMI is one of the most popular indicators for obesity because of its simple and non-invasive procedure. Many studies have investigated a possible link between obesity and periodontal disease. It is postulated that adipose tissue produces a vast number of hormones and pro-inflammatory cytokines called adipokines or adipocytokines such as interleukin- 6 and tumor necrosis factor- $\alpha$ which mediates endotoxin induced injury in various organs including periodontal tissues and results in periodontal destruction $[12,13]$.

There is increasing concern about the triangular relationship between diabetes, obesity, and periodontitis. In our study, the gingival status was assessed by gingival index. The mean gingival index score in group 1, group 2, and group 3 were $1.58,1.54$, and 1.25 respectively. Gingival health was poor among obese and non-obese diabetic patients (Groups 1 and 2) when compared with obese nondiabetic patients (Group 3). Our results were similar to the results reported by Arrieta-Blanco et al., in 2003 [10], Khader et al., in 2008 [8], and Kathiresan et al., in 2017 [2], who showed that gingival index score was significantly higher in diabetic than in non-diabetic subjects.
In this present study, periodontal status was assessed by community periodontal index by the presence of periodontal pockets and clinical loss of attachment. There was increased periodontal pockets in diabetic obese group (15.4\%), followed by diabetic non obese (4.66\%), and nondiabetic obese (2\%) group respectively. Our study results were similar to the findings observed by Arrieta-Blanco et al., in 2003 [10], Campus et al., in 2005 [14], Khader et al., in 2008 [8], Preshaw et al., in 2010 [9], and Kathiresan et al., in 2017 [2].

Increased glucose in saliva boosts bacterial growth and plaque formation. Glucose level in GCF of diabetes mellitus patients is two times higher compared to other patients. This high glucose level in GCF may lead to changes in bacterial composition in plaque and will affect periodontal disease [1,5].

In the present study, it was found that the loss of attachment was seen in all the three groups, but we found increased severity of loss of attachment in group 1 (60.7\%), followed by group $2(45.9 \%)$ and group $3(15.3 \%)$ respectively. Severe loss of attachment was found among obese diabetic patients. Similar findings were reported by Arrieta-Blanco et al., in 2003 [10], Campus et al., in 2005 [14], and Khader et al., in 2008 [8] who stated that loss of attachment was significantly higher in diabetic than in nondiabetic subjects.

Our findings indicate that periodontal health was poor in obese diabetic group compared to the obese nondiabetic group. Similar results were reported by Pham and Tran in 2017 [16] in Vietnamese population, and Samira et al., in 2008 [17] in Iranian population.

\section{Conclusion}

Both Type II diabetes and obesity affect the periodontium through the immune responses from the pro inflammatory cytokines such as tumor necrosis factor (TNF) and interleukins-6 (IL-6). The synergistic effect of these two conditions may lead to the higher risk of gingivitis and periodontitis in subjects who are diabetic as well as obese. This study substantiates the hypothesis that "patients who are obese with type II diabetic mellitus are at more risk in developing gingivitis and periodontitis which may result in compromised oral status". Hence, the risk of poor periodontal health in obese diabetic patients should be addressed earlier to prevent further complications and to achieve a good oral health status. There should be awareness about this triangular relationship among the rural and remote health care workers of our country. Increased cooperation between medical and dental professionals is important in reducing the global burden of disease and maintaining patients' optimal health. To the best of our knowledge, this is the very first study carried out in the Indian population to evaluate the gingival and periodontal status in obese and non-obese type II DM patients. 


\section{Acknowledgement}

We would like to thank the prestigious Indian Council of Medical Research for selecting this study (Reference ID 2019-07947) under Short Term Studentship (ICMR-STS) category and encouraging with scholarship. The authors are grateful to Dr. S. Prabhu, Associate Professor, Department of Public Health Dentistry, Asan Memorial Dental College and Hospital, in helping out with data analysis for this study.

\section{References}

1. Agarwal R, Baid R. Periodontitis and diabetes: A bidirectional, cyclical relationship - A brief review. Acta Med Int. 2017;4:46-49.

2. Kathiresan TS, Masthan KMK, Sarangarajan R, Babu NA, Kumar P. A Study of Diabetes Associated Oral Manifestations. J Pharm Bioallied Sci. 2017;9(Suppl 1):S211-S216.

3. Grossi SG, Genco RJ. Periodontal disease and diabetes mellitus: a two-way relationship. Ann Periodontol. 1998;3:51-61.

4. Awad M, Rahman B, Hasan H, Ali H. The Relationship between Body Mass Index and Periodontitis in Arab Patients with Type 2 Diabetes Mellitus. Oman Med J. 2015;30:36-41.

5. Malone JI, Hansen BC. Does obesity cause type 2 diabetes mellitus (T2DM)? Or is it the opposite? Pediatr Diabetes. 2019;20:5-9.

6. Wood N, Johnson RB, Streckfus CF. Comparison of body composition and periodontal disease using nutritional assessment techniques: Third National Health and Nutrition Examination Survey (NHANES III). J Clin Periodontol. 2003;30:321-327.

7. Levine RS. Obesity, diabetes and periodontitis--a triangular relationship? Br Dent J. 2013;215:35-39.
8. Deshpande K, Jain A, Sharma R, Prashar S, Jain R. Diabetes and periodontitis. J Indian Soc Periodontol. 2010;14:207212.

9. Khader YS, Albashaireh ZS, Hammad MM. Periodontal status of type 2 diabetics compared with nondiabetics in north Jordan. East Mediterr Health J. 2008;14:654-661.

10. Preshaw PM, de Silva N, McCracken GI, Fernando DJ, Dalton CF, Steen ND, et al. Compromised periodontal status in an urban Sri Lankan population with type 2 diabetes. J Clin Periodontol. 2010;37:165-171.

11. Arrieta-Blanco JJ, Bartolomé-Villar B, Jiménez-Martinez E, Saavedra-Vallejo P, Arrieta-Blanco FJ. Dental problems in patients with diabetes mellitus (II): gingival index and periodontal disease. Med Oral. 2003;8:233-247.

12. Mathur LK, Manohar B, Shankarapillai R, Pandya D. Obesity and periodontitis: A clinical study. J Indian Soc Periodontol. 2011;15(3):240-244.

13. Pataro AL, Costa FO, Cortelli SC, Cortelli JR, Abreu MH, Costa JE. Association between severity of body mass index and periodontal condition in women. Clin Oral Investig. 2012;16:727-734.

14. Campus G, Salem A, Uzzau S, Baldoni E, Tonolo G. Diabetes and periodontal disease: a case-control study. J Periodontol. 2005;76:418-425.

15. Maulani WK, Gartika M, Hendiani I. The difference of the gingival condition between diabetes mellitus and non diabetes mellitus patient on children at Cipto Mangunkusumo Hospital Jakarta. Padjadjaran Journal of Dentistry 2009;21:47-50.

16. Pham TAV, Tran TTP. The interaction among obesity, Type 2 diabetes mellitus, and periodontitis in Vietnamese patients. Clin Exp Dent Res. 2018;4:63-71.

17. Hajimaghsoodi S, Ghadiri-Anari A, Rashidi-Meybodi F, Jafarzadeh Z. Evaluation of CPITN and BMI Indices in Patients with Uncontrolled Type 2 Diabetes. Iranian Journal of Diabetes and Obesity. 2017;9:164-169. 